Probiotics and Microbiome

\title{
Respiratory and Gut Microbiota in Allergy and Asthma
}

\author{
Mirjana Turkalj ${ }^{1,2,3}$, Marcel Lipej ${ }^{4}$
}

${ }^{1}$ Srebrnjak Children's Hospital, Zagreb, Croatia, Department of Pediatric Allergology and Pulmonology, ${ }^{2}$ Josip Juraj Strossmayer University of Osijek, School of Medicine, Osijek, Croatia, ${ }^{3}$ Catholic University of Croatia, Zagreb, Croatia, ${ }^{4}$ Srebrnjak Children's Hospital, Zagreb, Croatia, Department of Translational Medicine

\section{Correspondence: turkalj@bolnica-srebrnjak.hr Tel.: + 38516391210 \\ Fax.: + 38516391188}

Received: January 14, 2019

Accepted: February 5, 2019

Key Words: Asthma - Childhood Microbiome - Gut Microbiota • Lung Microbiota.

\begin{abstract}
The objective of the study was to assess the potential mechanisms by which the microbiome influences immune responses in allergy and asthma, especially during early childhood and to evaluate the evidence of respiratory and gut microbiota dysbiosis. The development and progression of allergy and asthma may depend on individual susceptibility, allergen exposure, infections, and exposure to indoor and outdoor air pollution. In recent years there has increasingly been an emphasis on the importance of microbiota in the regulation of immune response and the development of atopic diseases, as well as asthma. Commensal microbes are necessary for the induction of a balanced and tolerogenic immune system especially at an early age. An insufficiency of early life exposure to the environmental microbiota necessary for colonization of the gastrointestinal and/or respiratory tracts results in the development of asthma and allergic diseases. On the other hand, disturbed physiological flora in the digestive and respiratory tract also increase the risk for development and progression of asthma and allergy (allergic march). Microbiota in the gut and lungs may influence both the onset and progression of childhood asthma. These microbiota can be modulated by various environmental factors, including the type of delivery, early-life microbial exposures, diet, antibiotics. Conclusion - Intervention regarding airway microbiome, particularly in early life, might be a strategy for prevention or treatment of asthma and allergies. Further investigations are needed to improve our understanding of the role of the microbiome in inflammation, and to clarify the effect of the regulation of lung and gut microbiota and its influence on the prevention or treatment of allergic diseases and asthma.
\end{abstract}

\section{Introduction}

Microbial communities have demonstrated unprecedented potential, in terms of their metabolic versatility, phyletic diversity and universality of habitats. It is indeed possible to say that microbial communities are truly ubiquitous, and as such are vital constituents of all aspects of the Earth's ecology, including interaction with humans. The interaction of humans with their native microbiota which is considered to be a supra organism or holobiont and is treated as an individual entity, is important in evolutionary selection. Consequently, the metabolic influence of microbial communities has a very direct influence on human and animal health. There is mounting evidence of the various levels of interaction between microbial communities and human hosts, especially those in the mucous tissues 
of the gastrointestinal and respiratory tracts $(1,2)$. This evidence becomes especially obvious when the microbial communities change or decrease in biodiversity, which is an effect caused by broad-spectrum antibiotics $(3,4)$. However, to date no clear mechanisms or specific metabolic circuits have been identified that are responsible for the interaction between microbial communities and human hosts. This hinders us from designing rational and targeted treatment options for various diseases related to microbial imbalances in humans, including asthma $(5,6)$. Diagnosis and treatment health disorders through microbial community modification e.g. with probiotics or symbiotics is therefore in its infancy and has so far been limited to a few cases, which rely more on phenomenological findings rather than a complete and thorough understanding of the actual contribution of microbial communities to the course and severity of a particular disease $(5,7)$. One of the rapidly increasing health problems where evidence exists for the interaction of human hosts and their indigenous microbiota are asthma and other allergic diseases (8). Among these, asthma is the most common chronic disease in children. Today half of Europe's population suffers from some form of allergy, and one person dies every hour from complications caused by allergies (9). In this asthma and allergy epidemic, there has been long-standing interest in the critical role of microbes in normal immune development and regulation.

This combination of a novel and multiple approach may lead to therapeutic opportunities associated with personalized microbialbased therapies in allergies and asthma.

\section{Microbiome and Immune Response}

Although it is known that the microbiome significantly influence host immune maturation and immune activity, the molecular basis for these immunomodulatory mechanisms are not clear. The immune system is composed of a complex network of innate and adaptive components, endowed with an extraordinary capacity to adapt and respond to highly diverse challenges, including microbiome dysbiosis. Alteration of the composition and function of the gut and/or respiratory microbiota as a result of antibiotic use or diet, and elimination of some pathogens such as helminth worms, has transformed our microbial allies into potential liabilities (8). Multiple mechanisms have been described through which bacteria can modulate immune and regulatory responses or the type of inflammation. The discoveries of molecular mechanisms, which can mediate host-microbe communication, have stressed the important role of microbiome in mucosal immune responses. Dendritic cells, $\mathrm{T}$ regulatory lymphocytes, B lymphocytes, NKT cells, ILCs and effector lymphocytes activities can all be modulated by the microbiome. Both bacterial strain components and microbiome metabolites have been associated with immune regulation in the gut or respiratory mucosa (10). Certain commensal microbes, such as specific Bifidobacterium and Lactobacillus strains, have been shown to increase the population of T regulatory cells (11), and can stimulate metabolic processes in dendritic cells, such as vitamin A metabolism, and tryptophan metabolism which promote $\mathrm{T}$ regulatory cells $(12,13)$. An exopolysaccharide from Bifidobacterium longum was found to suppress Th17 responses in the lungs and gut $(14,15)$. In addition, bacterialassociated components and bacterial-derived metabolites have a significant influence on immune regulatory processes. Short-chain fatty acids (acetate, butyrate and propionate) are produced by the gut microbiota and have been shown to modulate $\mathrm{T}$ cell and dendritic cell response, promoting epigenetic changes (16). Biogenic amine, synthetized due to 
amino acid metabolism from gut bacteria, may also modulate immune and inflammatory responses (17).

\section{Microbiota and Risk of Asthma in Childhood}

The first years of our lives are the critical period for susceptibility to environmental exposure as the immune system develops through complex host-environment inter actions (18). The human microbiome includes as many as $10^{14}$ bacteria, similar to the number of cells in an individual person $(1,2)$. The composition of the gut microbiota matures within the first years of life (18) and the microbiome may have the ability to cause host immune maturation, and may be an important environmental factor that determines the transition from health to disease. Perturbation of the microbiome during this critical period of development may cause asthma, allergy, and other immunological disorders $(19,20)$. In babies and infants, the presence of pathogenic bacteria in the lungs and gut has been associated with subsequent development of allergic sensitization and asthma (21). Furthermore, it has been observed that one-year-old children with an immature microbial composition have an increased risk of asthma at five years of age (22). Adequate maturation of the gut microbiota in this period may protect these pre-disposed children from asthma development (23). Birth by cesarean section has been recognized as a risk factor for asthma and other immune-mediated diseases in childhood. Furthermore, antibiotic exposure in the first year of life has also been associated with increased asthma risk, as it changes the microbial community (24). Actually, the lack of maturation of the microbiome in the first year of life is critical for increased asthma risk $(21,25)$. Manipulation of the airway microbiome, particularly in early life, might be a strategy to prevent or treat asthma, although the results of studies on prebiotics used together with probiotics have been negative overall. Probiotic intervention trials in pregnancy and early life have failed to prove efficacy in reducing asthma risk $(26,27)$. However, this could be due to species selection or the inadequate species composition of the probiotics. It may be speculated that the global microbial population and delayed maturation in the first year of life matters more than individual taxa.

\section{Gut Microbiota and Asthma}

The human gut in otherwise healthy subjects is colonized by $10^{14}$ bacteria and contains more than 1000 bacterial species $(1,2)$. The interaction of the human gut and its microbiome is conditioned by dietary and environmental variations, leading to the selection of a high variety of bacteria, interacting both for defense and nutritional purposes. The gut microbiota and their metabolic products exist in a dynamic state, which varies throughout the lifetime of the individual. This is particularly true during the first 18 months of life, as the gastrointestinal tract becomes colonized, and communities of bacteria form in various niches throughout the gut $(3,4,6$, 7). There is a growing appreciation of the fact that gut microbiota plays a vital role in host physiology as well as in the pathophysiology of several disorders, including asthma $(6,28)$. Using fecal samples from one year old babies, the microbial composition was analyzed and followed up in these babies up to school age (29). The results showed significant differences in microbial composition in children that had developed asthma, compared to those who had not. This difference was associated with whether the mother had asthma, indicating that these children are at a higher risk of developing asthma $(1,28)$.

Metagenomic studies of healthy and diseased individuals have revealed that reduced 
biodiversity and alterations in the composition of the gut are associated with asthma and other allergic diseases. For example, a relative decrease in the proportion of Bifidobacteria and the concomitant increase in other enteric flora species in infancy have been linked to diseased states later in life. In addition, increased numbers of Escherichia coli and Clostridium difficile are associated with the development of atopic diseases, such as asthma (6, 30). Altered indigenous microbiota and the general microbial deprivation characterizing the lifestyle of urban people in affluent countries appear to be risk factors for immune dysregulation and impaired tolerance $(1,3)$. Physical inactivity, antibiotic over-use and a western diet poor in fresh fruit and vegetables further enhance the risk. All this may act in synergy with the dysbiosis and disbalance of the gut flora $(3,4,31)$. Studies of immigrants moving from non-affluent to affluent regions indicate that immune tolerance mechanisms can rapidly become impaired in microbepoor environments (32).

The concept of a "common mucosal immune system" rests on the premise that there is cross-talk between human mucosal compartments and that microbially driven differences in mucosal immune function may be shared across sites $(3,4)$. Once microbes enter a niche and become established, a balance must be struck that maintains functional homeostasis between microbiome indifferent compartments and hosts. We believe that gut and respiratory tract microbiota are closely related in the control of the development and progression of childhood asthma.

\section{Respiratory Tract Microbiota and Asthma}

Until recently, it was believed that the lungs were sterile or free from bacteria. Actually, no new study has found evidence of a sterile lung environment. It is known that the respiratory mucosa in healthy individuals is inhabited by niche-specific bacterial communities $(1,33)$. The highest densities of bacterial communities are found in the upper respiratory tract, reaching up to $10^{3}$ viable bacteria per nasal swab from the nasal cavity and nasopharynx, with up to $10^{6} / \mathrm{ml}$ viable cells from oropharynx lavages $(1,34,35)$. In the trachea and lungs, the estimated numbers of bacteria are lower, with approximately $10^{2}$ bacterial cells/ $\mathrm{ml}$ being found in bronchoalveolar lavages from healthy lungs (36). The six dominant bacterial lines routinely found in the lung are Bacteroides, Fusobacteria, Proteobacteria, Acidobacteria, Actinobacteria and Furmicutes $(37,38)$. The composition of the respiratory tract microbiota develops exponentially very early in life, and may be influenced later in life by age, health status and environment. Type of delivery (vaginal or via caesarean section), exposure during the first hours of life and the environment of the following 3-4 months of life have been found to be most important in forming stable respiratory and gut microbiota, which are crucial to later health (39-41).

The different composition of the lung microbiota between asthmatic and healthy individuals indicates that bacteria may contribute to asthma development and suggests the important effect of immune responses for microbiota which inhabit organ systems, such as the gut $(1,7,32)$. This has led to the concept of the "gut-lung axis" $(1,2,7$, 40). In asthmatic airways there is an increase Proteobacteria phylum, including families of Oxalobacteraceae, Comamonadaceae, Nitrosomonadaceae, Sphingomonadaceae and Pseudomonadaceae (1, 42). Other studies also confirmed that Proteobacteria were present in greater numbers in asthmatic airways (36, 42). In patients with severe asthma a relationship between the airway microbiome dysbiosis and disease features has been found (Fig. 1.) $(43,44)$. Moreover, different clinical 


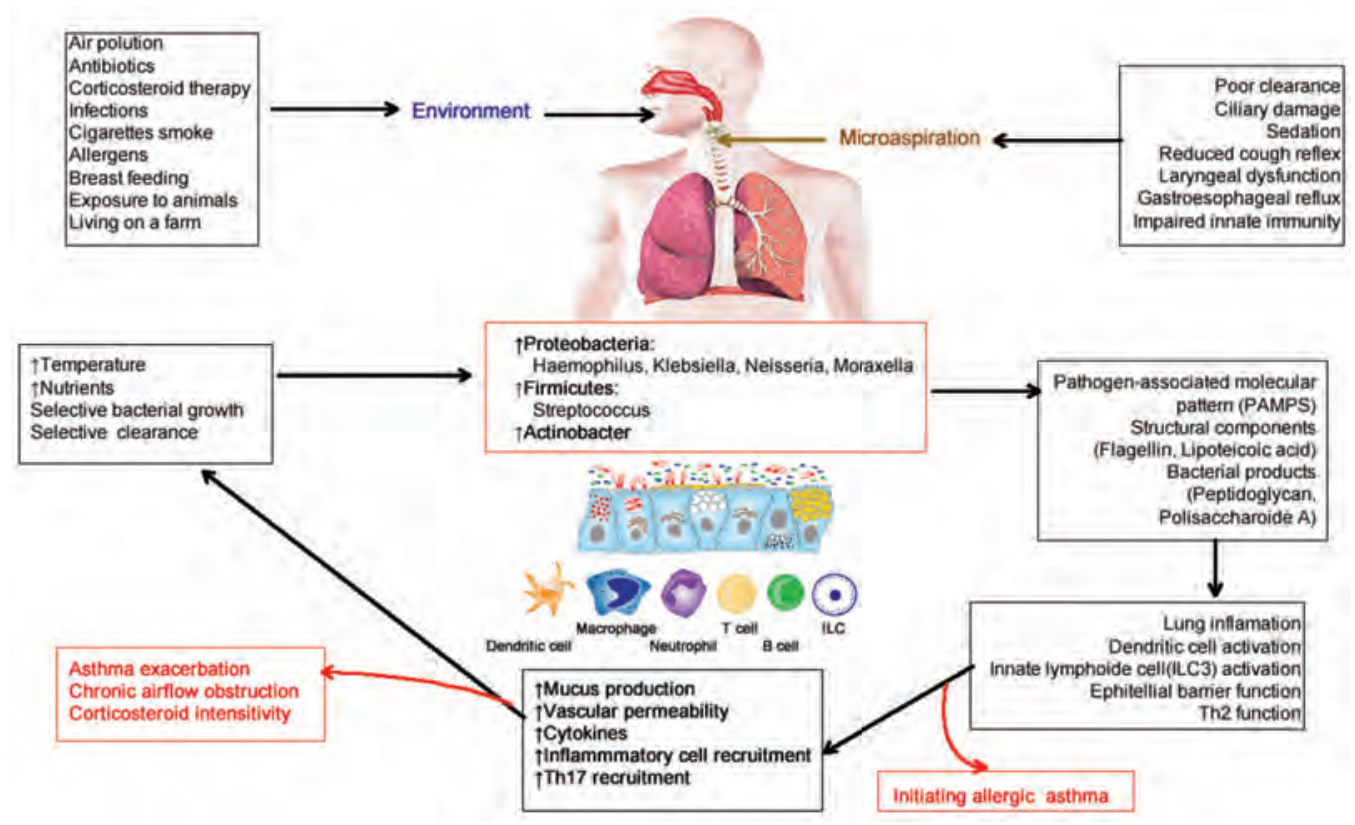

Fig. 1. Lung microbial dysbiosis in asthma (44).

phenotypes of severe asthma have been described, suggesting the possible involvement of microbiome dysbiosis in the modulation of inflammation in asthmatic airways (7, 45). Analysis of lower airway microbiota in asthmatic patients, poorly controlled despite high-dose of inhaled corticosteroid therapy, noted significant relationships between different bacterial communities. In fact, the species Klebsiella was augmented in patients with severe asthma in comparison with patients with mild-to-moderate asthma and controls $(43,46)$. In severe asthmatics Proteobacteria was associated with the $\mathrm{T}_{\mathrm{H}} 17$-related gene signature in airway epithelium, thereby worsening asthma control. Moreover, Bacteroides and Firmicutes phylum were more abundant in obese patients with severe asthma, whereas the presence of Actinobacteria correlated with improvement of asthma control (43). It has is also been shown that the species Neisseria, Haemophilus, Campylobacter and Leptotrichia were present in the lower airways of patients with corticosteroid-resistant asthma, but not in patients with corticosteroid-sensitive asthma (36). In severe asthmatics the presence of Mycoplasma pneumoniae and Chlamydophila pneumoniae, was found in several clinical trials, suggesting the validity of therapy with macrolide antibiotics $(38,45$, 47). It seems that microbiome-related functions might affect responsiveness to corticosteroid treatment in asthmatics. In patients with asthma in which Haemophilus levels were increased, a diminished response to corticosteroid therapy was found (48).

\section{The Role of Microbiome in Prevention and Treatment of Asthma}

At present, the available asthma treatments work to reduce and control symptoms but are unable to prevent and/or cure the diseases effectively. Recent studies on asthma have suggested possible therapeutics that may modulate innate immunity and microbiota (49). The effect of gut microbiota maturation over the first year of life in infants at high risk for asthma was investigated, alongside the fact that it is modifiable by Lactobacillus 
supplementation in early life (49). The study showed that early-life gut microbial development is distinct but plastic, which opens the possibility of a novel strategy for early-life prevention of childhood asthma. Considering the fact that manipulation of the gutairway axis and microbiome, particularly in early life, might be a strategy to prevent the development of asthma.

The reparation of lung and gut microbiota through the use of probiotics, prebiotics or symbiotics is one of the potential therapeutic strategies in asthmatics. Interest in this is relatively recent, especially based on evidence that a "healthy" microbial community facilitates the development of immune tolerance $(27,50)$. In animal models a study confirmed the protective effects of certain probiotic strains on inflammatory responses in lungs, but also showed that not all probiotics have the same effect (51). Prevention and intervention studies in humans have shown inconsistent results. It is necessary to emphasize that comparisons between studies in humans are difficult, due to different populations, ages, study design, geographic locations, lifestyle factors, and the heterogeneity of the probiotics and/or prebiotics used (27). Lee SC, et al. in a randomized controlled trial using a combination of fish oils and vegetable extracts with a probiotic, found a significant improvement in pulmonary function and a significantly reduced need for short-acting bronchodilators and inhaled corticosteroids in asthmatic children, suggesting that a combination of multiple approaches may lead to measurable clinical benefits (52). These results are promising, however more clinical studies are needed to determine whether modification of gut and lung microbiota can modulate the natural course of asthma, as well as controlling the disease (53). Currently, there is no recommendation to use pre- or probiotics for treatment or prevention of asthma (3). There is also no recommenda- tion regarding whether maternal diet during pregnancy and breastfeeding, with the addition of prebiotics or probiotics, could influence the development of allergy and asthma later in life (3). New studies are required to determine if appropriate prebiotic and probiotic use during pregnancy may functionally impact the risk of asthma and allergy in their children $(3,54)$. Further profiling and characterization of the microbiome associated with specific asthma phenotypes are necessary for identifying novel microbiota-related mechanisms of disease and also "phenotypespecific" management strategies (55).

\section{Conclusions}

The function of microbiota in healthy immune response is generally acknowledged, and respiratory and gut dysbiosis might result in chronic inflammatory disorders, such as asthma. Further investigations are needed to improve our understanding of the role of the microbiome in inflammation and its influence on the risk factors for asthma. In the last few years many studies have been published which prove the connection between the respiratory and gut microbiota and the development and treatment of asthma. The identification of novel bacterial strains and a combination of multiple approaches may lead to measurable clinical benefits and open new possibilities in the personalized prevention and treatment of asthma patients.

Conflict of Interest: The authors declare that they have no conflict of interest.

\section{References}

1. Huttenhower C, Gevers D, Knight R, Abubucker S, Badger JH, Chinwalla AT, et al. Structure, function and diversity of the healthy human microbiome. Nature. 2012;486:207-14.

2. Tap J, Mondot S, Levenez F, Pelletier E, Caron C, Furet JP, et al. Towards the human intestinal mi- 
crobiota phylogenetic core. Environ. Microbiol. 2009;11:2574-84.

3. Huang Y, Marsland B, Bunyavanich S, O’Mahony L, Leung D, Muraro A, et al. The microbiome in allergic disease: current understanding and future opportunities-2017 PRACTALL document of the American Academy of Allergy, Asthma \& Immunology and the European academy of allergy and clinical immunology. J Allergy Clin Immunol. 2017;139:1099-110.

4. Wong EH, Porter JD, Edwards MR, Johnston SL. The role of macrolides in asthma: current evidence and future directions. Lancet Respir Med. 2014;2:657-70.

5. Stefka AT, Feehley T, Tripathi P, Qiu J, McCoy K, Mazmanian SK, et al. Commensal bacteria protect against food allergen sensitization. ProcNatlAcadSci U S A. 2014;111:13145-50.

6. Cho I, Blaser MJ. The human microbiome: at the interface of health and disease. Nat Rev Genet 2012;13:260-70.

7. Stiemsma, LT,Turvey, SE. Asthma and the microbiome: Defining the critical window in early life. Allergy Asthma Clin. Immunol. 2017;13:3.

8. Prescott SL. Early-life environmental determinants of allergic diseases and the wider pandemic of inflammatory noncommunicable diseases. J Allergy Clin Immunol. 2013;131:23-30.

9. ISAAC Steering Committee. World wide variations in the prevalence of asthma symptoms: The International Study of Asthma and Allergies in Childhood (ISAAC). Eur. Respir. J. 1998;12:31535.

10. Tang C, Kamiya T, Liu Y, Kadoki M, Kakuta S, Oshima K, et al. Inhibition of Dectin-1 signaling ameliorates colitis by inducing lactobacillus-mediated regulatory $\mathrm{T}$ cell expansion in the intestine. Cell Host Microbe. 2015;18:183-97.

11. Konieczna P, Ferstl R, Ziegler M, Frei R, Nehrbass $\mathrm{D}$, Lauener RP, et al. Immunomodulation by Bifidobacteriuminfantis 35624 in the murine lamina propria requires retinoic acid-dependent and independent mechanisms. PLoS One. 2013;8:e62617. doi: 10.1371/journal.pone.0062617

12. Konieczna P, Groeger D, Ziegler M, Frei R, Ferstl R, Shanahan F, et al. Bifidobacterium infantis35624 administration induces Foxp3 T regulatory cells in human peripheral blood: potential role for myeloid and plasmacytoid dendritic cells. Gut. 2011;61:354-66.
13. Dasgupta S, Erturk-Hasdemir D, Ochoa-Reparaz J, Reinecker H, Kasper D. Plasmacytoid Dendritic cells mediate anti-inflammatory responses to a gut Commensal molecule via both innate and adaptive mechanisms. Cell Host Microbe. 2014;15:413-23.

14. Altmann F, Kosma P, O’Callaghan A, Leahy S, Bottacini F, Molloy E, et al. Genome analysis and characterisation of the exopolysaccharide produced by Bifidobacteriumlongum subsp. longum $35624^{\mathrm{TM}}$ PLoS One. 2016;11:e0162983.

15. Schiavi E, Gleinser M, Molloy E, Groeger D, Frei R, Ferstl R, et al. The surface-associated exopolysaccharide of Bifidobacteriumlongum 35624 plays an essential role in dampening host Proinflammatory responses and repressing local T H 17 responses. Appl Environ Microbiol. 2016;82:7185-96.

16. Smolinska S, Groeger D, O’Mahony L. Biology of the microbiome 1: interactions with the host immune response. Gastroenterol Clin N Am. 2017;46:19-35.

17. Pugin B, Barcik W, Westermann P, Heider A, Wawrzyniak M, Hellings P, et al. A wide diversity of bacteria produce and degrade biogenic amines within the human gastrointestinal tract. MicrobEcol Health Dis. 2017;28:1353881.

18. Sevelsted A, Stokholm J, Bønnelykke K, Bisgaard H. Cesarean section and chronic immune disorders. Pediatrics. 2015;135:e92-e98.

19. Bisgaard H, Li N, Bonnelykke K, Chawes BL, Skov T, Paludan-Müller G, et al. Reduced diversity of the intestinal microbiota during infancy is associated with increased risk of allergic disease at school age. J AllergyClinImmunol. 2011;128:646-52.

20. Gensollen T, Iyer SS, Kasper DL, Blumberg RS. How colonization by microbiota in early life shapes the immune system. Science. 2016;352:539-44.

21. Stokholm J,Blaser MJ, Thorsen J, Rasmussen MA, Waage J, Vinding RK, et al. Maturation of the gut microbiome and risk of asthma in childhood. Nat Commun. 2018;13;9(1):704.

22. Turnbaugh PJ, Hamady M, Yatsunenko T, Cantarel BL, Duncan A, Ley RE, et al. A core gut microbiome in obese and lean twins. Nature. 2009; 457:480-84.

23. Bäckhed F, Roswall J, Peng Y, Feng Q, Jia H, Kovatcheva-Datchary $P$, et al. Dynamics and stabilization of the human gut microbiome during the first year of life. Cell Host Microbe. 2015;17:690-703.

24. Bokulich NA, Chung J, Battaglia T, Henderson N, Jay M, Li H, et al. Antibiotics, birth mode, and 
diet shape microbiome maturation during early life. SciTransl Med. 2016;8:343ra82-343ra82.

25. Yassour M, Vatanen T, Siljander H, Hamalaine AM, Harkonen T, Ryhänen, SJ, et al. Natural history of the infant gut microbiome and impact of antibiotic treatment on bacterial strain diversity and stability. SciTransl Med. 2016;8:343ra81343 ra81.

26. Gensollen T, Iyer SS, Kasper DL, Blumberg RS. How colonization by microbiota in early life shapes the immune system. Science. 2016;352:539-44.

27. Frei R, Akdis M, O’Mahony L. Prebiotics, probiotics, synbiotics, and the immune system: experimental data and clinical evidence. Curr Opin Gastroenterol. 2015;31:153-8.

28. Huang YJ, Boushey HA. The microbiome in asthma. J Allergy Clin Immunol. 2015;135:25-30.

29. Abrahamsson T, Jakobsson H, Andersson A, Björkstén B, Engstrand L, Jenmalm M. Low gut microbiota diversity in early infancy precedes asthma at school age. Clin Exp Allergy. 2014;44:842-50.

30. Penders J, Gerhold K, Thijs C, Zimmermann K, Wahn U, Lau S, et al. New insights into the hygiene hypothesis in allergic diseases: mediation of sibling and birth mode effects by the gut microbiota. Gut Microbes. 2014;5:239-44.

31. Wheeler ML, Limon JJ, Bar AS, Leal CA, Gargus $\mathrm{M}$, Tang $\mathrm{J}$, et al. Immunological consequences of intestinal fungal dysbiosis. CellHostMicrobe. 2016;19:865-73.

32. Arrieta MC, Stiemsma LT, Dimitriu PA, Thorson L, Russell S,Yurist-DoutschetS, et al. Early infancy microbial and metabolic alterations affect risk of childhood asthma. Sci Transl. Med. 2015;7:307ra152.

33. Bassis CM, Erb-Downward JR, Dickson RP, Freeman CM, Schmidt TM, Young VB, et al. Analysis of the upper respiratory tract microbiotas as the source of the lung and gastric microbiotas in healthy individuals. MBio. 2015;6:e00037.

34. Charlson ES, Diamond JM, Bittinger K, Fitzgerald AS, Yadav A, Haas AR, et al. Lung-enriched organisms and aberrant bacterial and fungal respiratory microbiota after lung transplant. Am J RespirCrit Care Med. 2012;186:536-45.

35. Man WH, de SteenhuijsenPiters WA, Bogaert D. The microbiota of the respiratory tract: gatekeeper to respiratory health. Nat Rev Microbiol. 2017;15:259-70.
36. Goleva E, Jackson LP, Harris JK, Robertson CE, Sutherland ER, Hall CF, et al. The effects of airway microbiome on corticosteroid responsiveness in asthma. Am J RespirCrit Care Med. 2013;188:1193-201.

37. Watson RL, de KoffEM, Bogaert D. Characterising the respiratory microbiome.Eur Respir J. $2018 \mathrm{Nov}$ 28. pii: 1801711 . doi: 10.1183/13993003.017112018.

38. Denner DR, Sangwan N, Becker JB, Hogarth DK, Oldham J, Castillo J, et al. Corticosteroid therapy and airflow obstruction influence the bronchial microbiome, which is distinct from that of bronchoalveolar lavage in asthmatic airways. J Allergy Clin Immunol. 2016;137:1398-405.

39. Teo SM, Mok D, Pham K, Kusel M, Serralha M, Troy N, et al. The infant nasopharyngeal microbiome impacts severity of lower respiratory infection and risk of asthma development. Cell Host Microbe. 2015;17:704-15.

40. Stiemsma LT, Turvey SE. Asthma and the microbiome: defining the critical window in early life. Allergy Asthma Clin Immunol. 2017;13:3.

41. Bisgaard H, Hermansen MN, Buchvald F, Loland L, Halkjaer LB, Bønnelykke K, et al. Childhood asthma after bacterial colonization of the airway in neonates. N Engl J Med. 2007;357:1487-95.

42. Marri PR, Stern DA, Wright AL, Billheimer D, Martinez FD. Asthma-associated differences in microbial composition of induced sputum. J Allergy Clin Immunol. 2013;131:346-52.

43. Huang YJ, Nariya S, Harris JM, Lynch SV, Choy DF, Arron JR, et al. The airway microbiome in patients with severe asthma: associations with disease features and severity. J Allergy Clin Immunol. 2015;136:874-84.

44. Chung KF. Airway microbial dysbiosis in asthmatic patients. J Allergy clinimmunol. 2017;139:1071-81.

45. Carr TF, Kraft M. Chronic infection and severe asthma. Immunol Allergy Clin N Am. 2016:36483-502.

46. Huang YJ, Charlson ES, Collman RG, ColombiniHatch S, Martinez FD, Senior RM. The role of the lung microbiome in health and disease. A National Heart, Lung, and Blood Institute workshop report. Am J RespirCrit Care Med. 2013;187:1382-7.

47. Wong EH, Porter JD, Edwards MR, Johnston SL. The role of macrolides in asthma: current evidence and future directions. Lancet Respir Med. 2014;2:657-70. 
48. Durack J, Lynch S, Nariya S, Bhakta N, Beigelman A, Castro M, et al. Features of the bronchial bacterial microbiome associated with atopy, asthma, and responsiveness to inhaled corticosteroid treatment. J Allergy Clin Immunol. 2017;140:63-75.

49. Durack J, Kimes NE, Lin DL, Rauch M, McKean $\mathrm{M}$, McCauley $\mathrm{K}$, et al.Delayed gut microbiota development in high-risk for asthma infants is temporarily modifiable by Lactobacillus supplementation. Nat. Commun. 2018;9:707.

50. Kitazawa H, Villena J. Modulation of Respiratory TLR3-Anti-Viral Response by Probiotic Microorganisms: Lessons Learned from Lactobacillus rhamnosus CRL1505. Front Immunol. 2014;5:201.

51. Lyons A, O’Mahony D, O’Brien F, MacSharry J, Sheil B, Ceddia M, et al. Bacterial strain-specific induction of Foxp3+ $\mathrm{T}$ regulatory cells is protective in murine allergy models. Clin Exp Allergy. 2010;40:811-19.
52. Lee SC, Yang YH, Chuang SY, Huang SY, Pan WH. Reduced medication use and improved pulmonary function with supplements containing vegetable and fruit concentrate, fish oil and probiotics in asthmatic school children: a randomised controlled trial. Br J Nutr. 2013;110:145-55.

53. Chu DM, et al. Maturation of the infant microbiome community structure and function across multiple body sites and in relation to mode of delivery. Nat Med 2017;23:314-26.

54. Gray L, O’Hely M, Ranganathan S, Sly P, Vuillermin $\mathrm{P}$. The maternal diet, gut bacteria, and bacterial metabolites during pregnancy influence offspring asthma. Front Immunol. 2017;8:365.

55. Skolewska M, Frei M Lunjani N, Akdis A, O’Mahony L. Microbiome and asthma. Asthma Res Pract. 2018;4:1-9. 